\title{
Identification of a subcentimeter pulmonary adenocarcinoma using intraoperative near-infrared imaging during video-assisted thoracoscopic surgery
}

Jane J. Keating, MD, Gregory T. Kennedy, BA, and Sunil Singhal, MD, Philadelphia, Pa

Intraoperative identification of subcentimeter pulmonary nodules is challenging by video-assisted thoracoscopic surgery (VATS) because the tactile sense of the surgeon is reduced and exposure is limited. ${ }^{1}$ Techniques are available to aid in the detection of these nodules, including intraoperative pulmonary ultrasound, radionucleotide imaging, and computed tomography (CT)-guided and spiral wire localization. However, these technologies are accompanied by challenges of their own, most notably the need for prior information on the location of the nodule. ${ }^{2}$ We propose using

From the Division of Thoracic Surgery, University of Pennsylvania School of Medicine, Philadelphia, Pa.

Disclosures: Authors have nothing to disclose with regard to commercial support.

Received for publication Aug 25, 2014; revisions received Oct 15, 2014; accepted for publication Oct 16, 2014.

Address for reprints: Jane J. Keating, MD, Division of Thoracic Surgery, Hospital of the University of Pennsylvania, 6 White Building, 3400 Spruce St, Philadelphia, PA 19104 (E-mail: Jane.Keating@uphs.upenn.edu).

J Thorac Cardiovasc Surg 2015;149:e53-5

$0022-5223 / \$ 36.00$

Copyright (C) 2015 by The American Association for Thoracic Surgery

http://dx.doi.org/10.1016/j.jtcvs.2014.10.081 near-infrared (NIR) imaging during VATS to detect these nodules and resect them with negative margins. Our research focuses on intraoperative NIR imaging devices and fluorescent contrast agents, including indocyanine green (ICG), for detection of cancer. We report the detection of a nonpalpable subcentimeter adenocarcinoma using NIR imaging during VATS.

\section{CLINICAL SUMMARY}

A 65-year-old woman presented for management of a right lower lobe (RLL) lung nodule. Chest CT demonstrated a 2.1-cm RLL lesion and a 6-mm ground-glass opacity in the right upper lobe (RUL). Fluorine-18 fluorodeoxyglucose positron emission tomography showed a standardized uptake value of 9.8 in the RLL lesion, and the RUL lesion was undetectable (Figure 1).

The patient was enrolled in an institutional review board-approved trial of intraoperative NIR imaging after informed consent for this novel experimental model. She received $5 \mathrm{mg} / \mathrm{kg}$ of ICG 24 hours before VATS. During surgery, an eighth intercostal VATS port and a muscle-sparing
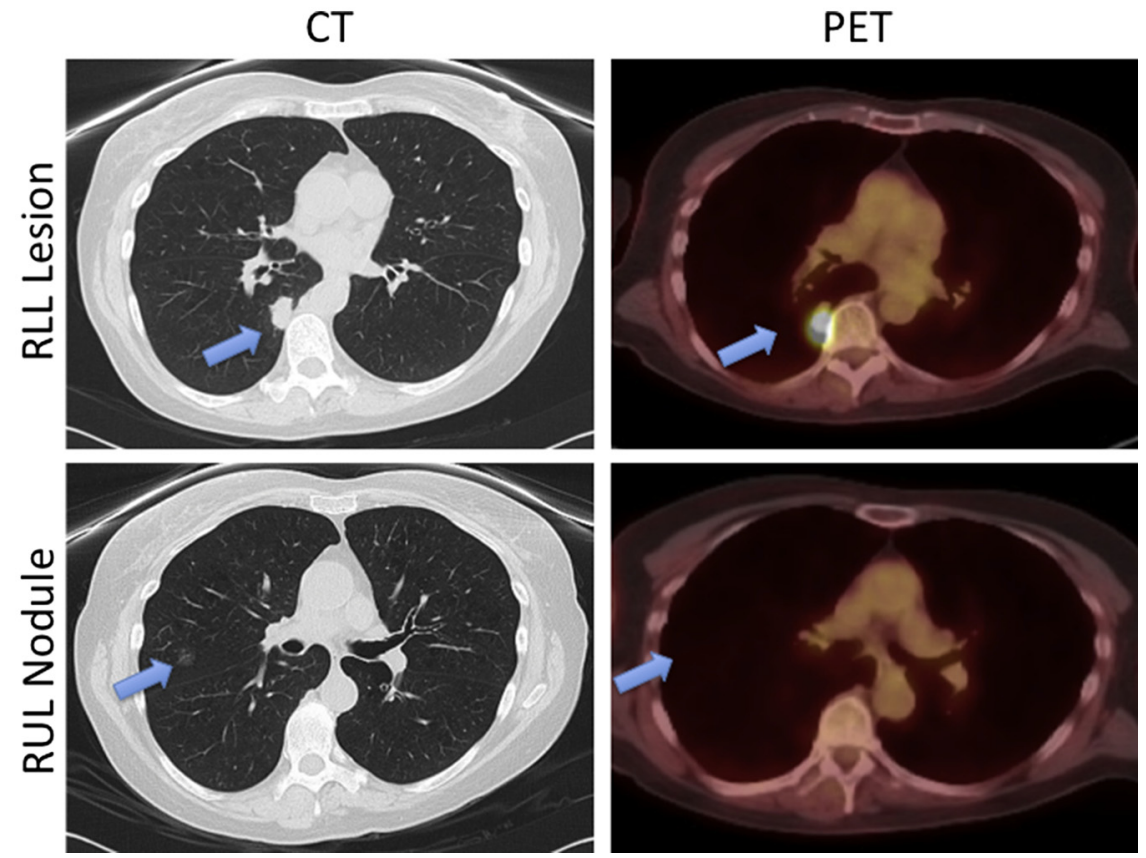

FIGURE 1. Preoperative CT scan demonstrating a 2.1-cm RLL nodule and a 6-mm RUL ground-glass opacity. Positron emission tomography scan of RLL nodule is positive ( 9.8 standardized uptake value), and RUL opacity is negative. $C T$, Computed tomography; PET, positron emission tomography; RLL, right lower lobe; $R U L$, right upper lobe. 
IN VIVO
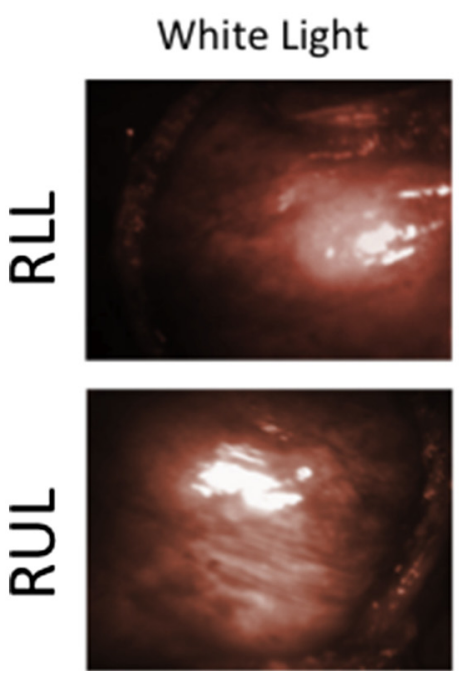

Fluorescence
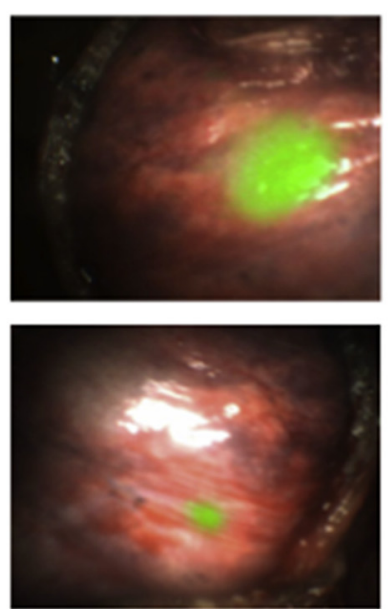

EX VIVO

White Light
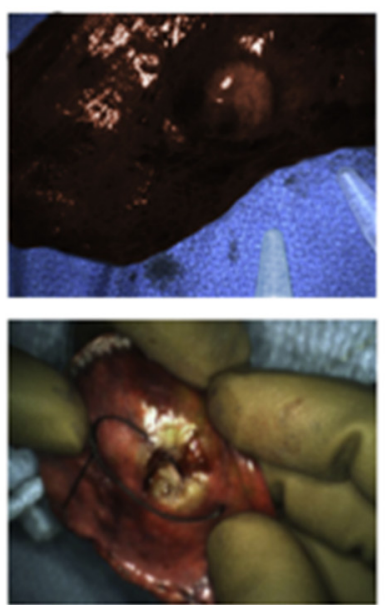

Fluorescence
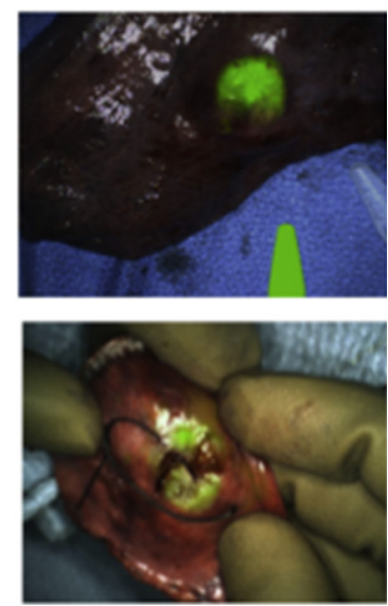

FIGURE 2. In vivo and ex vivo images of RLL lesion (top) and RUL nodule (bottom) including white light and fluorescence images taken with Artemis NIR imaging system (Quest Medical Imaging, Middenmeer, The Netherlands). RLL, Right lower lobe; RUL, right upper lobe.

incision were used for a planned VATS right lower lobectomy and RUL wedge. The dominant RLL lesion was visualized, but despite extensive manual palpation through both port sites, we could not detect the smaller RUL lesion. On examination with a NIR thoracoscope (Artemis; Quest Medical Imaging, Middenmeer, The Netherlands), there was a circumscribed area of fluorescence that was consistent with the opacity noted on preoperative CT scan. A wedge excision was performed using a NIR thoracoscope to monitor the location of the nodule and identify margins surrounding the area of fluorescence. The sample was inspected on the back table, and no lesion could be palpated or visualized under white light. When imaged, a strong fluorescent signal was noted. Postoperatively, a signal-to-background ratio of 3.6 was calculated using ImageJ digital analysis software (Figure 2). Frozen section confirmed adenocarcinoma, and the surgical margins were negative. The remainder of the operation included a right lower lobectomy and lymph node dissection. Again, on back-table analysis, the larger lesion was fluorescent with a signal-to-background ratio of 3.7. Final pathology indicated 2 primary stage I lung cancers: an 0.8-cm RUL minimally invasive adenocarcinoma and a 2.1-cm RLL poorly differentiated invasive squamous cell carcinoma. All lymph nodes were negative.

\section{DISCUSSION}

Annually, 80,000 people in the United States undergo surgery for a solitary lung nodule, and up to $15 \%$ of these patients have additional nodules not detected preoperatively. ${ }^{3}$ Intraoperatively, surgeons use VATS for visual inspection and finger palpation to search for these lesions. If necessary, the surgeon will typically convert to thoracotomy or perform a lobectomy for diagnosis.
This case demonstrates the use of NIR imaging for the detection of a subcentimeter pulmonary nodule. The tumor was less than $0.5 \mathrm{~cm}$ from the lung surface, but was neither visible nor palpable by VATS. The use of fluorescence imaging spared the patient a thoracotomy and the associated morbidities. Of note, both of the patient's tumors, 1 squamous cell carcinoma and 1 adenocarcinoma, were detectable with ICG. The detection of multiple cancer types confirms research in animal models showing ICG tumor localization in several murine carcinoma cell lines. ${ }^{4,5}$

NIR imaging has multiple advantages over other technologies for intraoperative pulmonary nodule detection. ${ }^{2}$ NIR imaging does not require radiation or require prior information about the nodule location, and can image a wide field, unlike other intraoperative imaging techniques. Finally, NIR imaging provides real-time information to the observer. Limitations of this technique include the potential for false-positive and negative fluorescence, as well as limitations to tissue penetration. Future research should focus on determining sensitivity and specificity for detecting lung cancer subtypes, as well as the minimum size and maximum depth of detectable tumor.

\section{CONCLUSIONS}

This case report demonstrates the successful use of NIR imaging during VATS in the detection of a subcentimeter nodule that was not identifiable by conventional visualization and manual palpation. In addition, we have had consistent success with this technique in several other patients with larger primary lung tumors. Improvement of this technology during minimally invasive surgery may allow for improved oncologic outcomes through earlier detection and resection of small malignant 
pulmonary nodules without the need to convert to lobectomy or open thoracotomy.

\section{References}

1. Parsons AM, Detterbeck FC, Parker LA. Accuracy of helical CT in the detection of pulmonary metastases: is intraoperative palpation still necessary? Ann Thorac Surg. 2004;78:1910-8.

2. Eichfeld U, Dietrich A, Ott R, Kloeppel R. Video-assisted thoracoscopic surgery for pulmonary nodules after computed tomography-guided marking with a spiral wire. Ann Thorac Surg. 2005;79:313-7.
3. Cerfolio RJ, McCarty T, Bryant AS. Non-imaged pulmonary nodules discovered during thoracotomy for metastatectomy by lung palpation. Eur J Cardiothorac Surg. 2009;35:786-91

4. Madajewski B, Judy BF, Mouchli A, Kapoor V, Holt D, Wang MD, et al. Intraoperative near-infrared imaging of surgical wounds after tumor resections can detect residual disease. Clin Cancer Res. 2012; 18:5741-51

5. Schaafsma BE, Mieog JS, Hutteman M, van der Vorst JR, Kuppen PJ Lowik CW, et al. The clinical use of indocyanine green as a near-infrared fluorescent contrast agent for image-guided oncologic surgery. J Surg Oncol. 2011;104:323-32.

\title{
Successful recovery from respiratory failure by external distraction sternoplasty in a patient with Jeune syndrome
}

\author{
Samina Park, MD, Chang Hyun Kang, MD, PhD, In Kyu Park, MD, PhD, and Young Tae Kim, MD, PhD, \\ Seoul, Republic of Korea
}

Jeune syndrome (JS), known as asphyxiating thoracic dystrophy, is a rare autosomal recessive disorder, reported by de Vries and colleagues ${ }^{1}$ in 1945. Abnormal endochondral bone formation causes a small, narrow, and noncompliant thorax. Pulmonary complications resulting from the restrictive thoracic cage are the primary cause of death, and up to $80 \%$ of the children with JS die as a result

From the Division of Thoracic Surgery, University of Pennsylvania School of Medicine, Philadelphia, Pa.

Disclosures: Authors have nothing to disclose with regard to commercial support. Received for publication July 22, 2014; revisions received Oct 29, 2014; accepted for publication Nov 6, 2014; available ahead of print Dec 10, 2014.

Address for reprints: Chang Hyun Kang, MD, PhD, Department of Thoracic and Cardiovascular Surgery, Seoul National University Hospital, 101 Daehak-ro, Jongno-gu, Seoul 110-744, Republic of Korea (E-mail: chkang@ snu.ac.kr). J Thorac Cardiovasc Surg 2015;149:e53-5

$0022-5223 / \$ 36.00$

Copyright (C) 2015 by The American Association for Thoracic Surgery http://dx.doi.org/10.1016/j.jtcvs.2014.11.030 of recurrent pulmonary infection. ${ }^{1}$ Herein, we report a case of a patient who successfully recovered from respiratory failure after thoracic cage enlargement, through gradual external distraction of the sternum.

The diagnosis of JS was made at a gestational age of 22 weeks. The patient was born at full term via spontaneous vaginal delivery; however, he was diagnosed with recurrent pulmonary infection and respiratory distress and was repeatedly admitted to the intensive-care unit. At the age of 7 months, he underwent tracheostomy and was dependent on a mechanical ventilator. At the age of 22 months, he was transferred to our institute for surgical correction of restrictive chest. His height and chest circumferences were below the 25th percentile for his age. The chest computed tomography scan revealed a narrow bell-shaped thoracic cage, which induced extrinsic compression of the left main bronchus, atelectasis, and hypoinflation of both lungs. The venous blood gas analysis

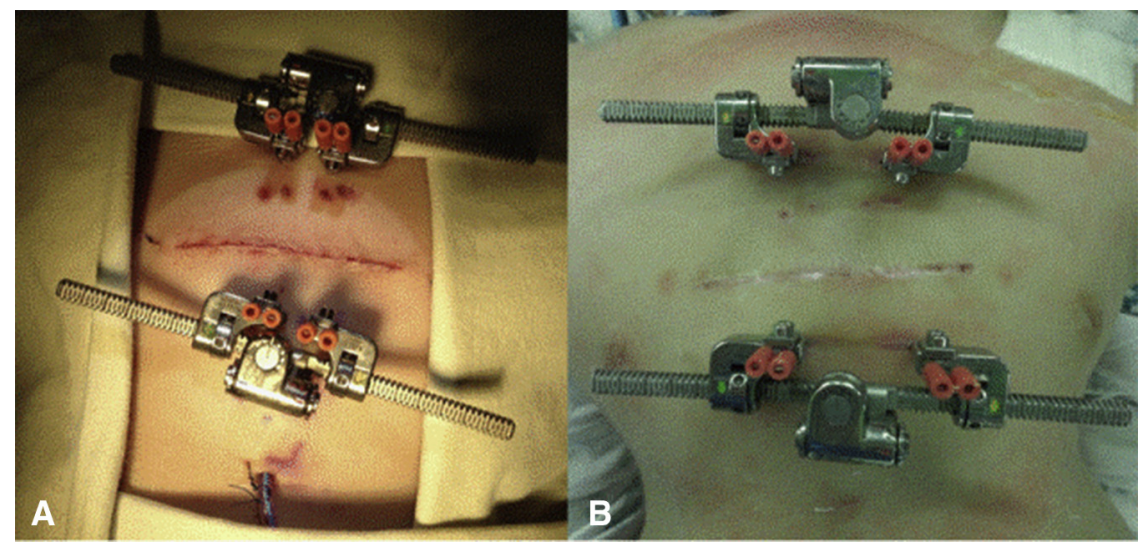

FIGURE 1. The figure shows (A) a photograph taken immediately after surgery; and (B) a photograph taken at postoperative week 3. 